\title{
Improved Frequency Offset Estimation Based on the DRM System
}

\author{
Wen Cui $\mathbb{D}$, Bo Li $\mathbb{D}$, and Jiaxu Zhang $\mathbb{D}$ \\ School of Communication and Information Engineering, Xi'an University of Posts and Telecommunications, Xi'an 710121, China \\ Correspondence should be addressed to Wen Cui; 1801200015cw@stu.xupt.edu.cn
}

Received 20 July 2020; Revised 6 April 2021; Accepted 15 April 2021; Published 5 May 2021

Academic Editor: Jun Cai

Copyright $\odot 2021$ Wen Cui et al. This is an open access article distributed under the Creative Commons Attribution License, which permits unrestricted use, distribution, and reproduction in any medium, provided the original work is properly cited.

\begin{abstract}
In this paper, an improved frequency offset estimation algorithm is proposed. It can effectively utilize pilot symbols in orthogonal frequency division multiplexing (OFDM) signals based on the Digital Radio Mondiale (DRM) system. In order to reduce the computational difficulty, the difference values of adjacent pilot symbols in the DRM system are sequentially combined into a pilot difference set. The pilot difference set is analyzed by inductive statistics so that the frequency offset estimator has higher calculation efficiency and accuracy. Through the induction statistics of multiple groups of symbols, the contingency of results can be effectively avoided. By comparing the performance of the proposed frequency offset estimator with that of the traditional estimators, the proposed algorithm has higher frequency offset estimation accuracy.
\end{abstract}

\section{Introduction}

The system named as Digital Radio Mondiale (DRM) originally mainly used a digital radio broadcasting specification below $30 \mathrm{MHz}$ [1]. As an important standard, DRM has been widely used in digital broadcasting. In terms of the transmitter, DRM has high quality within a given bandwidth, allowing more stations to use various MPEG-4 audio coding formats [2].

The DRM system multiplexes three logical channels, that is, the fast access channel (FAC), the main service channel (MSC), and the service description channel (SDC) [3]. FAC is the channel for multiplexing data stream that contains the information which begins decoding the multiplex and is necessary to find the services. The FAC is distributed across the transmission frames. The FAC block contains parameters describing multiple services along with a cyclic redundancy check (CRC) as well as parameters describing channels. MSC is a multichannel data stream channel that occupies the main part of the transmission frame and carries all digital audio services, as well as possible support and additional data services. SDC is the channel for the multiplexed data stream that provides information for decoding and the services contained in the multiplex. And each DRM transmission frame includes pilot cells, data cells, and control cells [4]. Pilot cells include time references, frequency references, and gain references.

Due to its outstanding spectrum utilization efficiency, orthogonal frequency division multiplexing (OFDM) has been successfully adopted by multitudinous wireless communication systems on frequency-selective fading channels [5]. Using OFDM modulation makes it possible to implement single-frequency networks (SFN) in broadcast systems such as DRM [6].

In terms of a receiver, frequency offset has a crucial impact on the quality of the audio. It has occurred due to the Doppler effect, multipath phenomena, and noise interference [7]. To ensure reception performance, frequency offset estimation is necessary.

In general, carrier frequency offset estimation can be roughly divided into two parts: post-FFT integer frequency offset [8] and pre-FFT fractional frequency offset [9]. Based on a large amount of research on synchronization algorithms, it can be roughly divided into two categories: nondata-assisted and training sequence-assisted.

Aiming at the OFDM system of frequency-selective fading channels, Jiang et al. proposed a frequency offset estimator based on a frequency-domain training sequence [10]. Chin proposed a time-domain non-data-assisted maximum likelihood (ML) estimation method based on the 
Doppler frequency for OFDM on doubly selective fading channels [11].

Under the frequency-selective fading channel, the problem of sampling frequency offset estimation based on the OFDM broadcasting system was proposed by Jung and You [12]. C. Yan and M. Yan came up with an integer frequency offset estimation algorithm which exploits the correlation of frequency pilots to construct a novel angle vector [13]. The residual carrier frequency offset estimation scheme of the OFDM broadcasting system based on cyclic delay diversity was proposed by Shin and You [14]. However, there is still some residual frequency offset after adopting these methods and the performance is not satisfactory. There is still room for improvement in the accuracy of their estimation.

In this paper, based on the received audio data, the method of estimating the integer frequency offset by using an inductive method and calculating the fractional frequency offset by using the correlation of the pilots has been presented. In order to prove the effectiveness of this method, the mean square error (MSE) of each method is calculated and compared. Based on theoretical analysis and simulation, the proposed scheme is verified and it is proven that compared with the original methods, the calculation difficulty in this paper is significantly reduced and the accuracy is higher.

\section{The DRM System Model}

The DRM transmitting signal consists of a series of OFDM symbols, each of which contains a guard interval and a useful part of the symbol. Each symbol is the sum of $K$ sine wave parts equally spaced in frequency. Each sine wave section is called a "cell" and is transmitted at the corresponding carrier position with a given phase and amplitude. Each carrier is referenced by the index $k$, which belongs to the interval $\left[k_{\min }, k_{\max }\right]$ ( $k=0$ corresponds to the reference frequency of the transmitted signal) [15]. Table 1 shows the reference parameters of $k$.

Each transmission superframe consists of three transmission frames, including $N_{s}$ OFDM symbols. Each transmission frame has duration of $T_{f}$, and each one contains a set of $K$ carriers, which are transmitted as $T_{s}$. In the transmission frame, the symbol is numbered from 0 to $N_{s}-1$. For each reference cell, the defined amplitude and phase are transmitted. The complex cell value is obtained.

$$
c_{r, s, k}=a_{s, k} \cdot U_{s, k}
$$

where

$$
U_{s, k}=e^{j 2 \pi v_{1024}[s, k] / 1024},
$$

where $r$ represents the number of the transmission frame, $s$ indicates the OFDM symbol number, and $a_{s, k}$ is the amplitude, which selects one of the following values based on the robust mode adopted, $\{1, \sqrt{2}, 2\}$.
TABLE 1: Carrier numbers for each robust mode.

\begin{tabular}{lccccccc}
\hline \multirow{2}{*}{ Robustness mode } & Carrier & \multicolumn{6}{c}{ Spectrum occupancy $(\mathrm{kHz})$} \\
& & 4.5 & 5 & 9 & 10 & 18 & 20 \\
\hline \multirow{2}{*}{ A } & $k_{\min }$ & 2 & 2 & -102 & -114 & -98 & -110 \\
& $k_{\max }$ & 102 & 114 & 102 & 114 & 314 & 350 \\
$\mathrm{~B}$ & $k_{\min }$ & 1 & 1 & -91 & -103 & -87 & -99 \\
& $k_{\max }$ & 91 & 103 & 91 & 103 & 279 & 311 \\
$\mathrm{C}$ & $k_{\min }$ & - & - & - & -69 & - & -67 \\
& $k_{\max }$ & - & - & - & 69 & - & 213 \\
\hline
\end{tabular}

For time references and frequency references in robust modes $\mathrm{A}, \mathrm{B}$, and $\mathrm{C}$, the phases $v_{1024}[s, k]$ can be defined.

$$
v_{1024}[s, k]=v_{1024}[0, k]
$$

where the phases $v_{1024}[0, k]$ are specified in Table 2 [15].

For gain references, the phases are specified.

$v_{1024}[s, k]=\left(4 Z_{256}[n, m]+p W_{1024}[n, m]+12 p^{2}(1+s) \bmod 1024\right)$,

where

$$
\begin{aligned}
& n=s \bmod 3, \\
& m=\left\lfloor\frac{s}{3}\right\rfloor, \\
& p=\frac{k-1-2 n}{6},
\end{aligned}
$$

where $\lfloor\cdot\rfloor$ represents the largest integer less than or equal to its parameter.

Among them, the values of $W_{1024}$ and $Z_{256}$ are specified in Table 3.

Combining the above, the transmitted signal is represented by the following expression:

$$
x(n)=\operatorname{Re}\left\{e^{j 2 \pi f_{R} n} \sum_{r=0}^{\infty} \sum_{s=0}^{N_{s}-1} \sum_{k=k_{\min }}^{k_{\max }} c_{r, s, k} \psi_{r, s, k}(n)_{k}\right\},
$$

where $\operatorname{Re}\{\cdot\}$ refers to finding the real part of the complex number.

$$
\psi_{r, s, k}(n)=\left\{\begin{array}{l}
e^{j 2 \pi\left(k / T_{u}\right)\left(n-T_{g}-s T_{s}-N_{s} r T_{s}\right)}, \\
0,
\end{array}\right.
$$

where $f_{R}$ refers to the reference frequency of the radio frequency signal, $T_{u}$ represents the duration of a useful symbol, $T_{g}$ is the duration of the guard interval, and $T_{s}$ is the duration of a symbol. 
TABLE 2: Definition of phase parameters.

\begin{tabular}{lcc}
\hline Robustness mode & Carrier index, $k$ & Phase index, $v_{1024}[0, k]$ \\
\hline \multirow{3}{*}{ A } & 18 & 205 \\
& 54 & 836 \\
& 72 & 215 \\
\hline \multirow{3}{*}{ B } & 16 & 331 \\
& 48 & 651 \\
& 64 & 555 \\
\hline & 11 & 214 \\
C & 33 & 392 \\
& 44 & 242
\end{tabular}

TABle 3: Parameter definition of matrix $W_{1024}$ and matrix $Z_{256}$.

\begin{tabular}{cccccc}
\hline \multirow{2}{*}{$W_{1024}[n, m]=\{\quad 0}$, & 512, & 0, & $512\}$, \\
& $\{0$, & 512, & 0, & 512, & $0\}$, \\
$Z_{256}[n, m]=\{\quad\{512$, & 0, & 512, & 0, & $512\}\}$ \\
\hline & $\{0$, & 57, & 164, & 64, & $12\}$, \\
& $\{168$, & 255, & 161, & 106, & $118\}$, \\
& $\{25$, & 232, & 132, & 233, & $38\}\}$ \\
\hline
\end{tabular}

\section{The Conventional Frequency Offset Estimation Scheme}

In this section, the traditional algorithms that use the characteristics of the pilot symbols in the DRM system to estimate integer frequency offset and the ML method to estimate fractional frequency offset are mainly described.

\subsection{Conventional Scheme A}

3.1.1. Integer Frequency Offset Estimation. The conventional integral frequency offset estimation method utilizes the correlation between receiver symbols and time derivatives. The receiver uses frequency reference cells (FRCs) to detect the existence of the received signal and estimate its integer frequency offset. The frequency references can be used for various channel tracking processes [16].

A FRC is defined.

$$
F(k)=\sqrt{2} e^{j 2 \pi \theta(k) / 1024},
$$

where the power gain of all frequency reference cells should be $\sqrt{2}$ for robustness modes $\mathrm{A}, \mathrm{B}$, and $\mathrm{C}$ which is depicted in [15] and $2 \pi \theta(k) / 1024$ represents the phase rotation of the pilot cell.

An integer frequency synchronization algorithm for the DRM system based on frequency references is proposed. The algorithm is introduced below [17].

Firstly, the algorithm needs Fast Fourier Transform (FFT) operation to estimate the power spectrum of receiver symbols.

$$
R_{\mathrm{fft}}(k)=\frac{1}{N_{a}} \sum_{i=0}^{N_{a}}\left|\sum_{n=0}^{4 N_{s}-1} x\left(n+(L-i) N_{s}\right) e^{-j\left(2 \pi / 4 N_{s}\right) n k}\right|,
$$

where $N_{a}$ is the amount of spectra used for averaging, $N_{g}+N=N_{s}$, and $L$ represents the symbol index.

In the case of frequency offset, the peak value of a pilot symbol will be offset. The integer frequency offset can be obtained by the displacement detection of the pilot symbols.

$$
\Delta_{\text {int }}=\frac{f_{s}}{4 N_{s}} \arg \max _{d} \sum_{k} R_{\mathrm{fft}}\left(d+P_{\mathrm{fac}}(k)\right),
$$

where

$$
P_{\text {fac }}(k)=4 \cdot k \cdot\left(\frac{N_{g}}{N}+1\right)
$$

where $\Delta_{\text {int }}$ is the estimation of integer frequency offset, $f_{s}$ represents the sampling rate, and $d$ is the trial value of $\Delta_{\text {int }}$.

3.1.2. Fractional Frequency Offset Estimation. A very small residual frequency offset remains unchanged after the integer frequency offset is estimated by the pilot symbols inserted into the DRM signal subcarriers [18]. The most commonly used algorithm for fractional frequency offset estimation is the ML algorithm.

Assuming that integral frequency offset has been compensated, the remaining fractional frequency offset is temporarily set as $\Delta_{f}$. Since the pilot values are fixed in each symbol, the phase difference value of the corresponding pilot symbols of the two symbols is $2 \pi \Delta_{f} T$, where $T=T_{s}+T_{g}$ [19].

$$
E\left\{x(n) x^{*}(n+m)\right\}= \begin{cases}d_{s}^{2}+d_{w}^{2}, & m=0, \\ d_{s}^{2} e^{-j 2 \pi \Delta_{f}}, & m=N, n \in I, \\ 0, & \text { others }\end{cases}
$$

where $d_{s}^{2}$ and $d_{w}^{2}$ represent the energy of useful symbol and white Gaussian noise and $I$ is the cyclic prefix of the symbol $L$.

The estimated time delay is expressed as follows.

$$
\tau_{\mathrm{ML}}=\arg \max _{i}(|\gamma(i)-\rho| \phi(i)||),
$$

where

$$
\begin{aligned}
\gamma(i) & =\sum_{n=i}^{n=i+N_{g}-1} x(n) x^{*}(n+N), \\
\phi(i) & =\frac{1}{2} \sum_{n=i}^{n=i+N_{g}-1}\left(x^{2}(n)+x^{2}(n+N)\right), \\
\rho & =\frac{\mathrm{SNR}}{\mathrm{SNR}+1},
\end{aligned}
$$

where SNR is the Signal-to-Noise Ratio (SNR). Then, the fractional frequency offset can be obtained. 


$$
\Delta_{f}=-\frac{1}{2 \pi} \angle \gamma\left(\tau_{\mathrm{ML}}\right)
$$

where $\angle(\cdot)$ stands for angle operation.

By this traditional algorithm, the fractional frequency offset is obtained.

$$
\Delta=\Delta_{\text {int }}+\Delta_{f}
$$

where $\Delta$ is the total frequency offset of the final estimation.

The traditional method mainly uses the power factor of the pilot symbols to calculate the correlation. Due to noise and multipath interference, the power factor of the pilot symbols is inevitably affected so that the correlation peak may be weakened. Combined with the above analysis, the implementation of this algorithm is complicated and unstable.

3.2. Conventional Scheme B. In the case of frequency selectivity, a pilot-assisted-based estimation method is proposed [12]. The algorithm eliminates the need for channel state information by a two-step correlation and obtains unbiased estimation under frequency selective fading.

First of all, after FFT demodulation, the OFDM symbol received at the $k$ th subcarrier is obtained.

$$
R(k)=|H(k)|^{2}|x(k)|^{2} e^{\left(j 2 \pi \phi D_{t} N_{s} / N\right)}+I(k)+Z(k), \quad 0 \leq k<N,
$$

where

$$
\begin{aligned}
I(k)= & \sum_{n=0, n \neq k}^{N-1} H(n) x(n) e^{j \pi\left(2 \phi\left(N_{s}+N_{\mathrm{g}}\right) /(N+(N-1)(\phi+n-k) N)\right)} \\
& \cdot \frac{\sin (\pi(\phi+n-k))}{N \sin (\pi(\phi+n-k) / N)},
\end{aligned}
$$

where $H(k)$ is the frequency response of zero-mean channel with variance $\sigma_{H}^{2}, \phi$ is the frequency offset including carrier frequency offset and sampling frequency offset, $D_{t}$ is the distance between two continuous gain reference cells (GRCs) in the direction of time, and $Z(k)$ is a zero-mean complex Gaussian noise with variance $\sigma_{Z}^{2}$. Under the premise of not losing universality, it is assumed that GRCs of length $N_{p}$ are inserted symmetrically or uniformly at the subcarrier position $\left\{-k_{N_{p} / 2},-k_{N_{p} / 2-1}, \cdots,-k_{1}, k_{1}, \cdots, k_{N_{p} / 2}\right\}$. Thus, the estimation of frequency offset is obtained.

$$
\Delta=\frac{N}{2 \pi D_{t} N_{s}} \frac{\sum_{m=1}^{N_{p} / 2} \arg \left\{R\left(k_{m}\right) R^{*}\left(-k_{N_{p} /(2+1-m)}\right)\right\}}{\sum_{m=1}^{N_{p} / 2} k_{m}+k_{N_{p} /(2+1-m)}} .
$$

The above method is used to estimate the frequency offset of the system as $\Delta$. Conventional scheme $\mathrm{B}$ is aimed at improving the accuracy of frequency offset estimation in the case of frequency selective fading. This method estimates the sampling frequency offset and the carrier frequency offset as a whole, but there is still a small part of residue, and there is still room for improvement.

3.3. Conventional Scheme $C$. Traditional algorithm $\mathrm{C}$ uses the correlation of pilots to construct an angle vector for frequency offset estimation [13]. $M+1(M>1)$ consecutive received symbols are utilized by the algorithm. After two adjacent symbols are conjugate multiplied, the power spectrum $P_{i}(k)$ of the corresponding carrier is obtained.

$$
P_{i}(k)=X_{i}(k) X_{i+1}^{*}(k), \quad 0 \leq k<N, 0 \leq i<M,
$$

where $X(k)$ is the function of $x(n)$ after the Fourier transform.

The phase of the power spectrum is obtained.

$$
\mathrm{Ph}_{i}(k)=a c \tan \left(P_{i}(k)\right) .
$$

Define the angle vector as $\operatorname{Ang}(k)$.

$$
\operatorname{Ang}(k)=\sum_{i=0}^{M-1} \cos \left(\mathrm{Ph}_{i}(k)\right)+j \sum_{i=0}^{M-1} \sin \left(\mathrm{Ph}_{i}(k)\right)
$$

Thus, the frequency offset can be obtained.

$\Delta=\arg \max _{d}\{|\operatorname{Ang}(k 1+d)|+|\operatorname{Ang}(k 2+d)|+|\operatorname{Ang}(k 3+d)|\}, \quad 0 \leq d<N$,

where $k 1, k 2$, and $k 3$, respectively, represent the position of the frequency references. According to the characteristics of the DRM pilots, the frequency offset value $\Delta$ is estimated.

3.4. Conventional Scheme D. Traditional method D proposes a scheme to estimate frequency offset by cyclic delay and pilot mode [14]. Perfect symbol timing recovery is assumed the receiving end. In the case of frequency offset, obtain the $l$ th received symbol signal.

$$
R(k)=H_{l}(k) X_{l}(k) e^{j 2 \pi \Delta_{c}\left(l N_{s}+N_{g}\right) / N}+W_{l}(k),
$$

where $\Delta_{c}$ is the frequency offset normalized by the subcarrier spacing and $W_{l}(k)$ is the contribution of the zero-mean additive white Gaussian noise (AWGN) with variance $\delta_{W}^{2}$.

Supposing the channels $\left\{H_{l}^{t}(k), t=0,1\right\}$ are the frequency flat, the channel transfer function is decomposed.

$$
\begin{aligned}
\sum_{k \in S_{p}}\left|H_{l}(k)\right|^{2}= & \frac{N_{p}}{2}\left(\left|H_{l}^{0}(k)\right|^{2}+\left|H_{l}^{1}(k)\right|^{2}\right)+S\left(N_{p}\right) \sqrt{A^{2}+B^{2}} \cos \\
& \cdot\left(\frac{2 \pi\left(k_{1}+k_{N_{p}}\right) \sigma_{1}}{N}+\theta\right),
\end{aligned}
$$


with

$$
\begin{aligned}
& A=H_{l}^{0, I}(k) H_{l}^{1, I}(k)+H_{l}^{0, Q}(k) H_{l}^{1, Q}(k), \\
& B=-H_{l}^{0, I}(k) H_{l}^{1, I}(k)-H_{l}^{0, Q}(k) H_{l}^{1, Q}(k),
\end{aligned}
$$

where

$$
\begin{aligned}
S\left(N_{p}\right) & =\frac{\sin \left(\pi N_{p} D_{f} \sigma_{1} / N\right)}{\sin \left(\pi D_{f} \sigma_{1} / N\right)}, \\
\theta & =\tan ^{-1}\left(\frac{B}{A}\right),
\end{aligned}
$$

where $S_{p}$ is a set of GRC containing $N_{p}$ elements and $x^{I}$ and $x^{Q}$ represent the real and imaginary parts of $x$, respectively.

Then, use the GRC mode with the highest channel transfer function power to estimate the frequency offset.

$$
\widehat{l}=\arg \max _{l}\left\{\sum_{k \in S_{p}}\left|H_{l}(k)\right|^{2}\right\} .
$$

The maximum value of the channel transfer function can be approximated as the following formula:

$$
H_{\hat{l}}(k)=\sum_{k \in S_{p}}\left|H_{l \wedge}(k)\right|^{2} .
$$

The frequency offset is estimated.

$$
\Delta=\frac{E_{s} N_{p} H_{\hat{l}}(k)}{2} e^{j 2 \pi \Delta_{c} D_{t} \rho}+\sum_{k \in S_{p}} \widehat{W}_{\widehat{l}}(k),
$$

where $D_{t}$ is the periodicity of the GRC mode in the time domain and $\rho=\left(N_{g}+N\right) / N$.

This method selects the time delay by looking for the delay that makes the channel transfer function power different. Then, the frequency offset is estimated once in the four repeated pilot symbols to maximize the power of the channel transfer function. Therefore, these conditions are used to improve the frequency offset synchronization performance.

\section{The Proposed Frequency Offset Estimation Scheme}

In this part, an algorithm of frequency offset estimation based on inductive reasoning and correlation calculation of symbols is proposed. Among them, the integer frequency offset estimation is mainly based on the time reference values of the fixed positions in the standard. The fractional frequency offset is mainly estimated by the correlation of two adjacent symbols in the transmission frame.

4.1. Integer Frequency Offset Estimation. First of all, the transmission signal with time reference values is called the first
TABLE 4: The channel parameters.

\begin{tabular}{lcccc}
\hline Path & Property & Channel 1 & Channel 2 & Channel 3 \\
\hline \multirow{3}{*}{1} & Gain & 1 & 1 & 1 \\
& Delay (ms) & 0 & 0 & 0 \\
& Df (Hz) & 0 & 0 & 0 \\
\hline \multirow{3}{*}{2} & Gain & 0 & 1 & 1 \\
& Delay (ms) & 0 & 2 & 2 \\
& Df (Hz) & 0 & 0 & 1.2 \\
\hline \multirow{3}{*}{3} & Gain & 0 & 0 & 0.25 \\
& Delay (ms) & 0 & 4 & 4 \\
& Df (Hz) & 0 & 2 & 2.4 \\
4 & Gain & 0 & 0 & 0.00625 \\
& Delay (ms) & 0 & 0 & 6 \\
& Df (Hz) & 0 & 0 & 7.2 \\
\hline
\end{tabular}

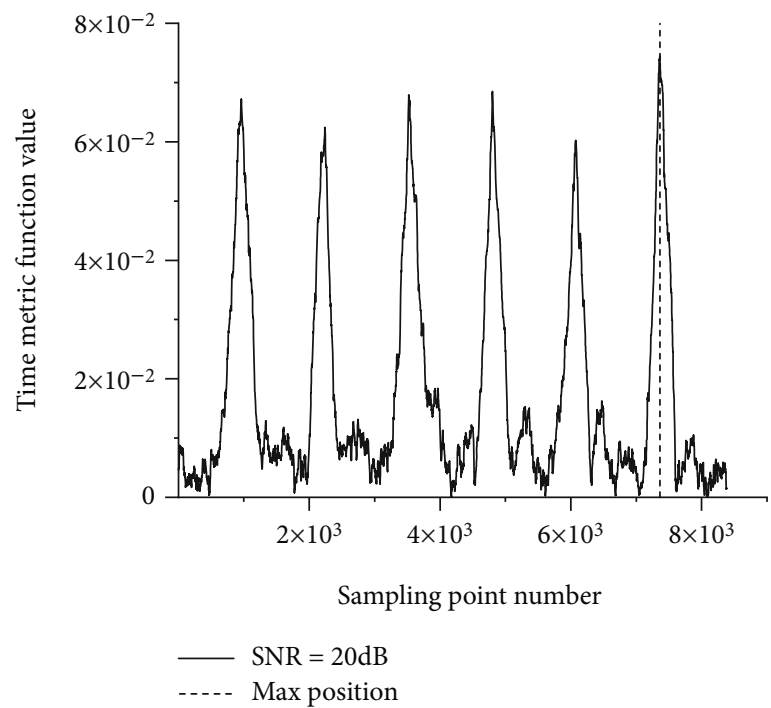

Figure 1: Timing synchronization accuracy.

signal of the transmission frame. The first signal is locked by the timing synchronization precision method.

$$
X_{\text {cor }}(n)=\sum_{l=0}^{N_{g}-1} x^{H}(n+l) \cdot x(n+l+N), \quad 1 \leq n \leq N_{l},
$$

where $X_{\text {cor }}$ represents the correlation between signals, $n$ is the index position in the OFDM signal, $H$ represents the conjugate transpose, and $N_{l}$ is the desired signal length.

$$
P_{\text {osi_max }}=\arg \max _{n}\left\{\left|X_{\text {cor }}(n)\right|\right\} \text {, }
$$

where $P_{\text {osi_max }}$ denotes the maximum position of the first signal.

The length of a signal is intercepted from its maximum position. The signal removed the guard interval and FFT operation is performed. 


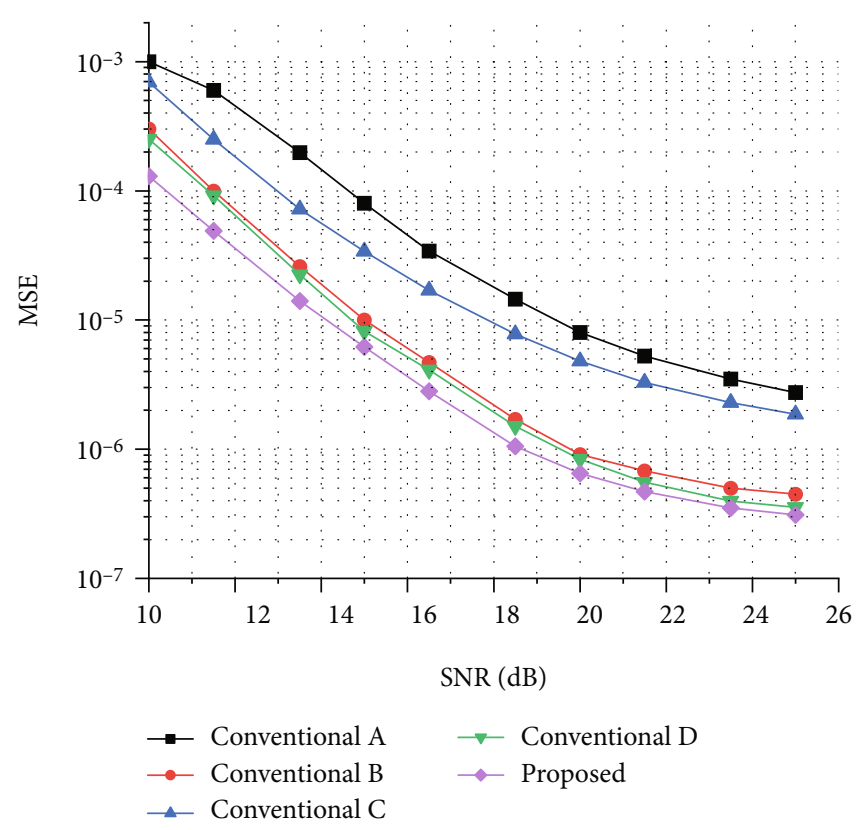

FIGURE 2: MSE of the conventional algorithms and the proposed algorithm in channel 1.

$$
\begin{aligned}
x_{\mathrm{ng}}(n) & =x(l), \quad P_{\text {osi_max }}+N_{\mathrm{g}}<l<P_{\text {osi_max }}+N_{s}, \\
X(k) & =\sum_{n=0}^{N-1} x_{\mathrm{ng}}(n) e^{-j 2 \pi k(n / N)},
\end{aligned}
$$

where $x_{\mathrm{ng}}$ represents the signal after removing the guard interval and $X(k)$ is the signal generated by FFT transformation.

$$
P_{i}(k)=\frac{\angle(X(k))}{2 \pi} * N
$$

where $P_{i}(k)$ is the value of the time reference after recovery.

Assume that $a, a+n, a+m \cdots$ are the correct positions of pilot symbols, respectively.

$$
D_{p}=\left\{P_{i}(a)-P_{i}(a+n), P_{i}(a)-P_{i}(a+m), \cdots\right\},
$$

where $D_{p}$ refers to the set of differences between the pilot symbols.

After integer frequency offset occurs, $e, e+n, e+m \cdots$ indicate the possible locations of pilot symbols.

$E_{p}(e)=\left\{P_{i}(e)-P_{i}(e+n), P_{i}(e)-P_{i}(e+m), \cdots\right\}, \quad 1 \leq e \leq N$,

where $E_{p}(e)$ represents the possible difference set of pilot symbols.

$$
B=\arg \min _{e}\left|D_{p}-E_{p}(e)\right|,
$$

where $B$ is the minimum set after the difference between the two difference sets.
Suppose that when $B$ reaches the minimum, its corresponding position of the first pilot signal is $h$.

$$
\Delta_{\text {int }}^{\prime}=|h-a| .
$$

By this inductive statistical method, the integer frequency offset is estimated.

4.2. Fractional Frequency Offset Estimation. Based on the position of the first signal obtained in the above section, the angular difference of each position corresponding to the guard interval between adjacent signals will be obtained.

$$
A(i)=\angle\left(x\left(P_{\text {osi_max }}+i\right) \cdot x^{H}\left(P_{\text {osi_max }}+i+N\right)\right),
$$

where $i$ stands for the position of the guard interval of the first symbol, whose scope is $0 \leq i \leq N_{g}-1$.

$$
\Delta_{f}^{\prime}=\frac{E[A(i)]}{2 \pi \cdot N},
$$

where $\Delta_{f}^{\prime}$ is the estimated fractional frequency offset.

$$
\Delta^{\prime}=\frac{\Delta_{\mathrm{int}}^{\prime}}{N}+\Delta_{f}^{\prime},
$$

where $\Delta^{\prime}$ is the normalized frequency offset obtained by the algorithm proposed in this paper.

Through the above method, the frequency offsets obtained by estimating multiple groups of symbols are sequentially placed in the set $\Delta^{\prime \prime}$. Average this set. 


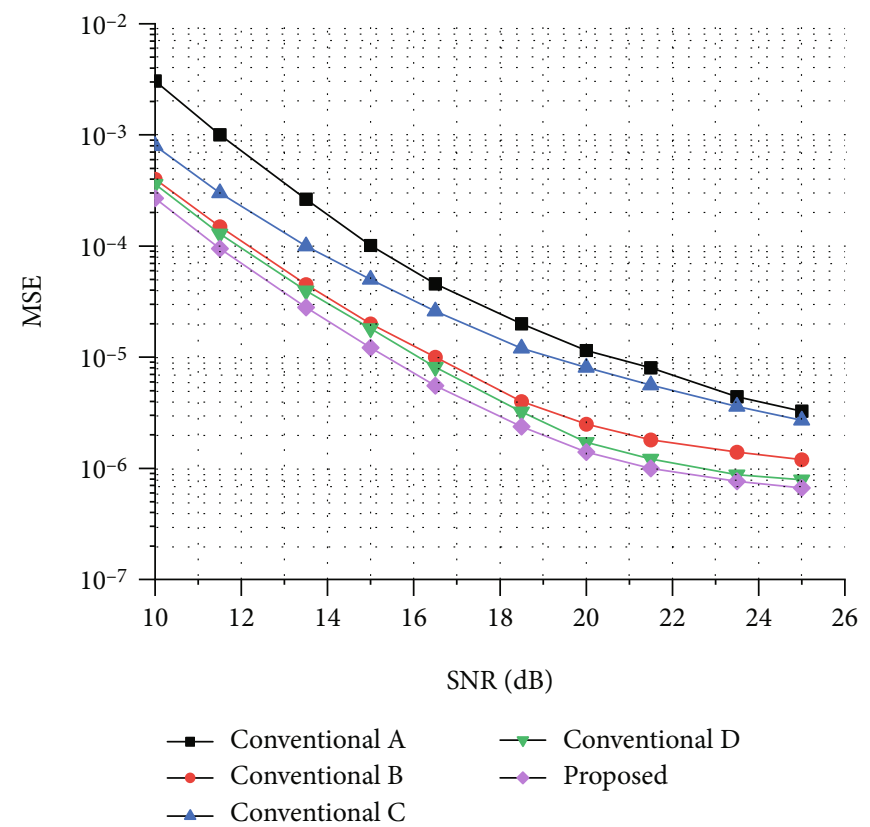

FIGURE 3: MSE of the conventional algorithms and the proposed algorithm in channel 2.

$$
\bar{\Delta}=E\left[\Delta^{\prime \prime}\right]
$$

where $\bar{\Delta}$ is the final frequency offset.

The previous algorithms only preserve the pilot phase factor in the correlation calculation. Considering the complexity of the algorithm, only a small number of continuous symbols are selected. The algorithm proposed in this paper integrates the differences between pilots into a set and estimates multiple groups of symbols, which greatly reduces the contingency of experimental results. With more continuous signals, the estimation accuracy is effectively improved.

In the case of a large calculation scale, the time complexity of the algorithm is calculated by $O(f(n))$, where $O(\cdot)$ represents the order of magnitude and $f(n)$ represents the function of the algorithm. Therefore, the time complexity of the algorithm proposed in this paper is $O\left(n^{2}\right)$. The time complexity of conventional scheme A is $O\left(n^{2}\right)$. The time complexity of conventional scheme $\mathrm{B}$ is $\mathrm{O}\left(n^{4}\right)$. The time complexity of conventional scheme $\mathrm{C}$ is $\mathrm{O}\left(n^{2}\right)$. The time complexity of conventional scheme D is $O\left(n^{2}\right)$. In terms of time complexity, the algorithm proposed in this paper is the same as conventional scheme A, conventional scheme $\mathrm{C}$, and conventional scheme D. However, in terms of the difficulty of coding the algorithm, the code of the algorithm proposed in this paper is easier to implement through formula analysis. In summary, through the comprehensive analysis of time complexity and difficulty of code writing, the algorithm proposed in this paper is more computationally efficient.

\section{Simulation Results}

In this section, simulations of the MSE of the estimated frequency offset under the different SNR and channel condi- tions will be obtained. The different parameters of the four paths contained in each channel are shown in Table 4.

All frequencies mentioned in the simulation experiment are normalized frequencies [20].

$$
\bar{\Delta}=\frac{\bar{\Delta}_{f} \times 2}{f_{s}},
$$

where $\bar{\Delta}_{f}$ is the actual frequency.

Figure 1 shows the time synchronization result obtained by Equation (37) and the highest position of the first signal obtained by Equation (38) under the condition that the frequency offset is normalized to 0.0313 . The highest position represents the position of the captured frame head. Thus, the signal of one symbol length is intercepted from the frame head position to estimate frequency offset.

Define the MSE for the frequency synchronization as $M$ $(\bar{\Delta})[21]$

$$
M(\bar{\Delta})=E\left[|\Delta \wedge-\bar{\Delta}|^{2}\right]
$$

where $\widehat{\Delta}$ is the actual frequency offset and $\bar{\Delta}$ is the frequency offset estimated by the proposed algorithm.

By introducing Equations (20), (23), (27), (36)), and (49) into Equation (51), respectively, the MSE of the two traditional algorithms and the method proposed in this article can be obtained under the corresponding channel environment. The results are shown in Figures 2-4.

Figures 2-4 show the comparison of the MSE between the proposed algorithm in this paper and the previous algorithms in channel 1, channel 2, and channel 3, respectively, under different SNR conditions. These simulations are carried out under the normalization of frequency offset to 


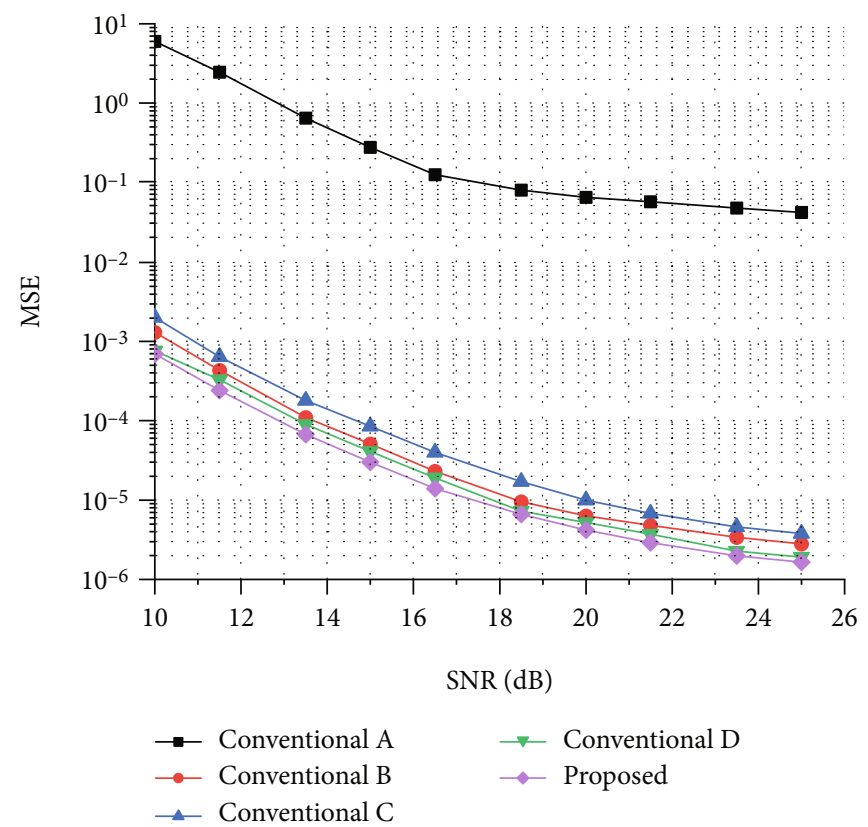

FIGURE 4: MSE of the conventional algorithms and the proposed algorithm in channel 3.

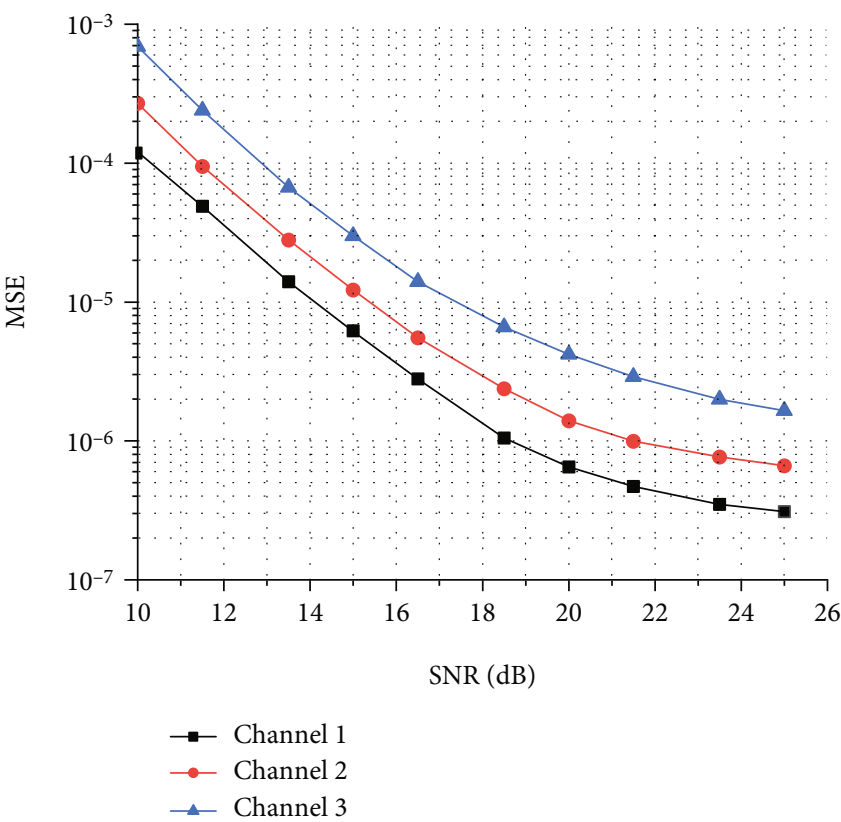

FIGURE 5: MSE of the proposed algorithm in each channel.

0.0313. As shown in Figure 2, in channel 1, the performance of the algorithm in this paper is at least about $0.6 \mathrm{~dB}$ higher than that of the original algorithms. As shown in Figure 3, in channel 2, the performance of the algorithm in this paper is at least about $0.6 \mathrm{~dB}$ higher than that of the original algorithms. As shown in Figure 4, in channel 3, the performance of the algorithm in this paper is at least about $0.6 \mathrm{~dB}$ higher than that of the original algorithms. Through the comparison of the three simulations, the MSE of the proposed algorithm is smaller than that of the previous algorithms under the cor- responding SNR in channel 1, channel 2, and channel 3. Under the same channel, the better the SNR condition, the higher the accuracy of the algorithm. The accuracy of the estimation is reduced to a certain extent by the relatively poor channel environment.

Through the above calculation method of MSE, Figure 5 shows the performance comparison of the MSE in three channels under the corresponding conditions of different SNR. This simulation is still carried out with the frequency offset normalized to 0.0313 . 
It can be concluded from the simulation diagram that the channel environment has a significant influence on the accuracy of frequency offset estimation. Obviously, under the same SNR, the better the channel environment, the smaller the MSE of the frequency offset estimation, which proves that the channel environment with better quality can improve the accuracy of frequency offset estimation to a certain extent.

\section{Conclusions}

In this paper, according to the characteristics of the inductive statistics method and pilot symbols, a frequency offset estimation algorithm for the DRM system is proposed. Then, this algorithm effectively utilizes the pilot phase factor. In the correlation calculation of fractional frequency offset estimation, the structure of an angle vector availably reduces the influence of multipath and noise. By comparing the MSE between the proposed algorithm and the traditional algorithms, the simulation results show that the proposed algorithm can more effectively improve the accuracy of frequency offset estimation.

\section{Data Availability}

Upon reasonable request and with the permission of all authors, the data used to support the algorithm results of this article can be obtained from the corresponding authors.

\section{Conflicts of Interest}

The authors declare that there is no conflict of interest in the publication of this paper.

\section{Acknowledgments}

This work was funded by the Shaanxi Province Science and Technology Coordination and Innovation Project (Key Technology (Chain) for Resource-Oriented Industries): High-Efficiency Multistandard Digital Broadcasting Pointer (Project Number 2011KTCL01-10).

\section{References}

[1] Y. H. You and K. T. Lee, "Accurate pilot-aided sampling frequency offset estimation scheme for DRM broadcasting systems," IEEE Transactions on Broadcasting, vol. 56, no. 4, pp. 558-563, 2010.

[2] M. Dietz and T. Mlasko, "Using MPEG-4 audio for DRM digital narrowband broadcasting," in 2000 IEEE International Symposium on Circuits and Systems. Emerging Technologies for the 21st Century. Proceedings (IEEE Cat No.00CH36353), Geneva, Switzerland, 2000.

[3] N. Zhu, Z. Li, and Q. Lu, "Research and implement of DRM baseband encoder and modulator based on embedded system," in 2010 Second International Conference on Networks Security, Wireless Communications and Trusted Computing, Wuhan, China, 2010.

[4] A. F. Kurpiers and V. Fischer, "Open-source implementation of a Digital Radio Mondiale (DRM) receiver," in Ninth International Conference on HF Radio Systems and Techniques, Bath, UK, 2003.
[5] A. Lipovac, V. Lipovac, and P. Njemčević, "Suppressing the OFDM CFO-caused constellation symbol phase deviation by PAPR reduction," Wireless Communications and Mobile Computing, vol. 2018, 8 pages, 2018.

[6] A. T. S. C. Standard, Physical Layer Protocol, Document A/322, ATSC, Washington, DC, USA, 2017.

[7] A. Kumar, M. Magarini, H. D. Joshi, and R. Saxena, "Exact SER analysis of DFrFT-based QPSK OFDM system over frequency selective Rayleigh fading channel with CFO," Journal of Computer Networks and Communications, vol. 2016, Article ID 2804507, 7 pages, 2016.

[8] D. Toumpakaris, J. Lee, and H.-L. Lou, "Estimation of integer carrier frequency offset in OFDM systems based on the maximum likelihood principle," IEEE Transactions on Broadcasting, vol. 55, no. 1, pp. 95-108, 2009.

[9] Y.-H. You, Y.-A. Jung, and J.-H. Paik, "Joint estimation of symbol timing and sampling frequency offset for CDDOFDM-based DRM systems," IEEE Transactions on Broadcasting, vol. 65, no. 2, pp. 333-339, 2019.

[10] Y. Jiang, X. Gao, and X. You, "Frequency offset estimation for OFDM systems with a novel frequency domain training sequence," IEICE Transactions on Communications, vol. E89B, no. 4, pp. 1194-1204, 2006.

[11] W.-L. Chin, "Non-data-aided Doppler frequency estimation for OFDM systems over doubly-selective fading channels," IEEE Transactions on Communications, vol. 66, no. 9, pp. 4211-4221, 2018.

[12] Y.-A. Jung and Y.-H. You, "Robust sampling frequency offset estimation for OFDM over frequency selective fading channels," Applied Sciences, vol. 8, no. 2, p. 197, 2018.

[13] C. Yan and M. Yan, "Integer frequency offset algorithm for Digital Radio Mondiale system," Journal of Communications, vol. 8, no. 9, pp. 572-578, 2013.

[14] W. J. Shin and Y. H. You, "A robust frequency offset estimation scheme for OFDM system with cyclic delay diversity," World Academy of Science, Engineering and Technology, vol. 6, no. 5, 2012.

[15] ETSI ES 201980, Digital Radio Mondiale (DRM): System Specification, European Telecommunications Standards Institute, 2017.

[16] Keukjoon Bang, Namshin Cho, Jaehee Cho et al., "A coarse frequency offset estimation in an OFDM system using the concept of the coherence phase bandwidth," IEEE Transactions on Communications, vol. 49, no. 8, pp. 1320-1324, 2001.

[17] V. Fischer and A. Kurpiers, "Frequency synchronization strategy for a PC-based DRM receiver," in 7th Int. OFDMWorkshop (InOWo'OZ), Hamburg, 2002.

[18] X. Jiang and Z. Dong, "Carrier frequency offset estimation of DRM receiver," in ICMMT 4th International Conference on, Proceedings Microwave and Millimeter Wave Technology, 2004, Beijing, China, 2004.

[19] A. Bo, "Fine decimal frequency offset and sampling clock offset estimation in HDTV," Journal of Southeast University (Natural Science Edition), vol. 33, no. 3, pp. 266-270, 2003.

[20] R. Sharma and N. Shrivastava, "Spectral efficiency and BER of OFDM systems with carrier frequency offset (CFO)," International Journal of Current Engineering and Technology, vol. 5, no. 6, 2015.

[21] W. J. Shin, J. Seo, and Y. H. You, "MSE analysis of sampling frequency offset estimation scheme for OFDM-based Digital Radio Mondiale (DRM) systems," Wireless Personal Communications, vol. 71, no. 2, pp. 1271-1281, 2013. 